\title{
TER: A System for Robotic Tele-echography
}

Adriana Vilchis Gonzales ${ }^{1}$, Philippe Cinquin ${ }^{1}$, Jocelyne Troccaz ${ }^{1}$, Agnès Guerraz ${ }^{2}$, Bernard Hennion $^{2}$, Franck Pellissier ${ }^{2}$, Pierre Thorel ${ }^{2}$, Fabien Courreges ${ }^{3,4}$, Alain Gourdon ${ }^{3}$, Gérard Poisson ${ }^{3}$, Pierre Vieyres ${ }^{3}$, Pierre Caron ${ }^{4}$, Olivier Mérigeaux ${ }^{4}$, Loïc Urbain ${ }^{4}$, Cédric Daimo ${ }^{5}$, Stéphane Lavallée ${ }^{5}$, Philippe Arbeille ${ }^{6}$, Marc Althuser ${ }^{7}$, Jean-Marc Ayoubi ${ }^{7}$, Bertrand Tondu ${ }^{8}$, Serge Ippolito ${ }^{8}$

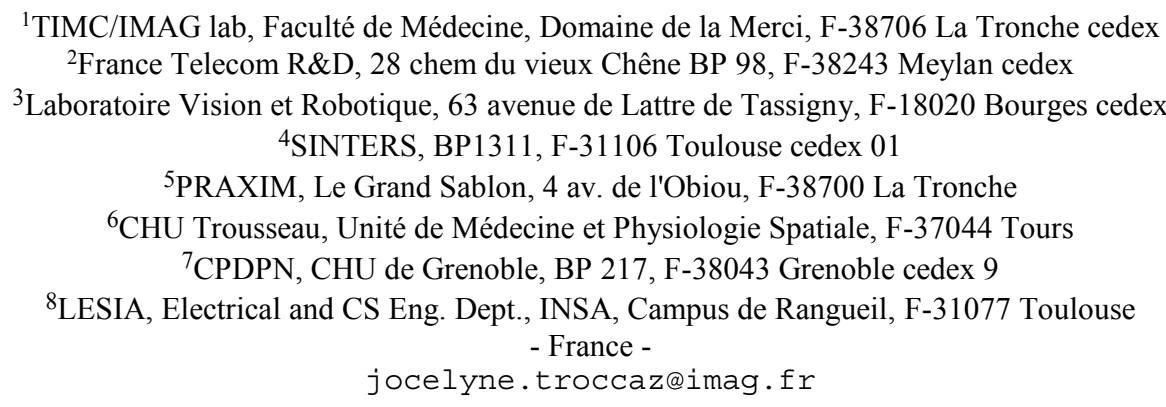

\begin{abstract}
The quality of ultrasound based diagnosis highly depends on the operator's skills. Some healthcare centres may not have the required medical experts on hand when needed and therefore may not benefit from highly specialized ultrasound examinations. The aim of this project is to provide a reliable solution in order to perform expert ultrasound examinations in distant geographical areas and for the largest population possible. TER is a tele-robotic system designed and developed by a French consortium composed of universities, hospitals and industrial companies. One originality of TER is the development of a compliant slave robot actuated by muscles. This slave robot is teleoperated by an expert clinician who remotely performs the exam. In this paper, we present the architecture of TER and describe its components.
\end{abstract}

\section{Introduction}

Echography is a difficult examination based on specialized skills. The clinician performing the examination moves the echographic probe on the patient's body to acquire noisy bi-dimensional images. He may perform his diagnosis from static measurements performed on these images and/or from a dynamical behaviour analysis of the organs for instance depending on the pressure exerted by the probe on the body. This is typically the case when diagnosing the presence of thromboses in the lower limbs. Therefore, performing such an examination involves a good eye-hand coordination and the ability to integrate the acquired information over time and space. Some of these highly specialized skills may lack in some healthcare centres or for emergency situations. Tele-consultation is therefore an interesting alternative to conventional care. 


\section{State of the Art}

Several projects have been launched worldwide for developing tele-echography. In France, the LOGINAT project [1] based on a multi-centres Visio-conferencing basis has been experimented since 1993 for inter-hospital perinatal care. The project evolved from real-time transmission of the exams to the transmission of static images and related documents. This evolution was due to the limited performance of communication networks at that time. The objective of the TeleinVivo European project [2] was to develop a portable station to allow echographic exams in isolated regions. With a 3D ultrasound probe, a volume of data can be sent to a remote expert who, using a virtual ultrasound sensor, can examine the data in much the same way that he would examine a patient.

Such projects are basically tele-medicine ones. The operator has still to perform the exam on the patient even if he can be guided by the expert; moreover the expert has to perform the diagnosis on a purely visual basis. From our point of view, a teleechography system has to integrate the ability for the expert to remotely move the echographic probe. The SYRTECH system [3,4] was developed in this spirit. The echographic probe is mounted on a 3 degree of freedom (dof) robot. This system is positioned manually on the patient by an operator. To explore the anatomical region of interest, the 3 rotations are remotely controlled by the expert using a virtual probe which motions are tracked by a magnetic localizer. SYRTECH has been demonstrated by performing from France remote exams on a man participating to a Nepalese expedition. Another system for US tele-intervention and laparoscopic ultrasound telemanipulation was studied within the MIDSTEP European project [5]. MIDSTEP integrates conventional robotic architectures. Salcudean [6] describes a light robot developed for the assistance of diagnostic ultrasound system; three control schemes have been implemented and tested: motion and force controls and visual servoing. Similarly, [7,8] present HIPPOCRATE, a hybrid force-position controlled robot for assisted echographic diagnosis; such a robot could potentially be integrated in a teleechography application. Finally, [9] proposes a very original parallel architecture for tele-robotic echography.

Most of these systems are still under development and have been partly validated. For some of them, a specific slave robot has been designed preferring safety and natural compliance to accuracy. This is acceptable because the accuracy requirements are generally lower for tele-robotic echography than for surgical applications; moreover, the operator closes the loop and may compensate for positional errors.

\section{Global Specifications and System Architecture}

Figure 1 illustrates the global architecture of TER. A virtual probe is mounted on the master interface device. The real probe is placed on the slave robot end-effector. Position and force information are transmitted bi-directionally. Live visual and audio data are also transmitted in both directions. The system is initialised to match the two environments. Then, mainly based on the echographic images and force information he receives back, the expert operator can move the virtual probe to control the real one. The slave robot executes the orders sent from the master site. A non expert op- 
erator is located close to the patient and supervises the procedure that he can interrupt. The patient can at any time communicate with him or with the expert. TER is designed to be used with low-bandwidth widespread networks in order to make it usable on a large scale.

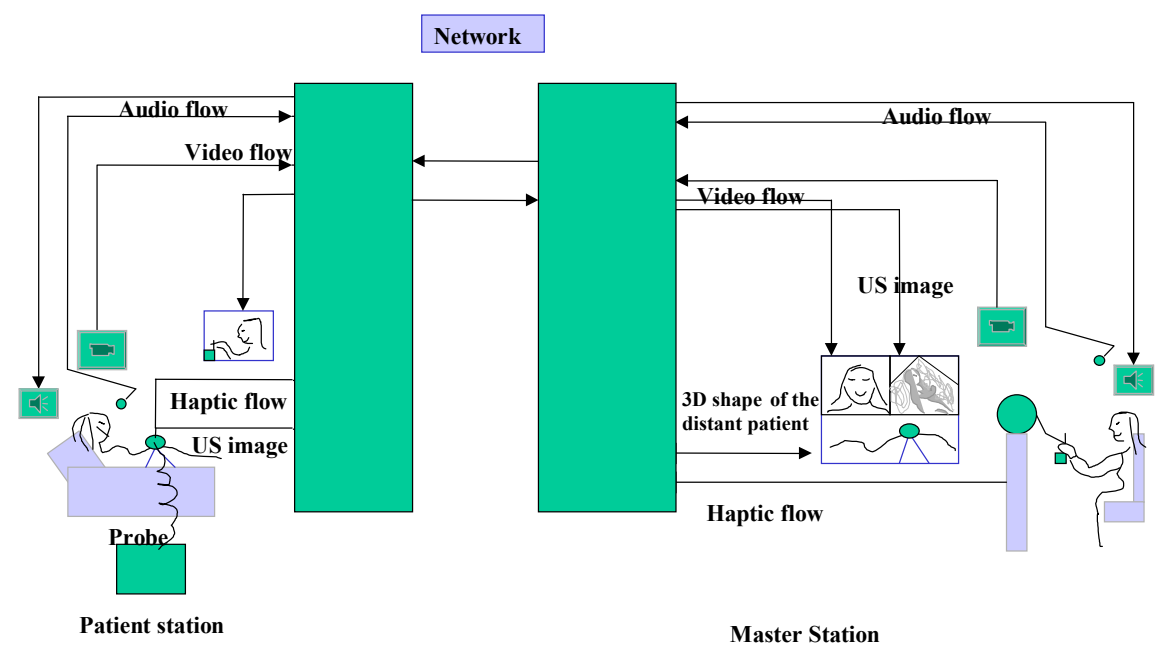

Fig. 1. TER general architecture

TER is under development. The following paragraphs describe the different components and their status of development.

\section{Master System}

To accurately reproduce the expert hand movement and the displacement of the ultrasound virtual probe, two solutions of different cost and complexity are being considered and tested. The first one uses a position sensor associated with visual feedback. The other one uses an haptic device that adds force feedback.

\section{Master Position-Based Interface Device}

In this version of the master interface device, a magnetic position device is used to track the virtual probe: the Flock of Bird (FOB) localizer from Ascension Technology. This magnetic tracker is a six degree-of-freedom measuring device that can be configured to simultaneously track position and orientation within a $\pm 130 \mathrm{~cm}$ range from the transmitter. A sensor is affixed to the probe. 
A second version of this position-based interface system is currently designed in order to integrate a one-direction force feedback rendering to the distant expert the pressure exerted back from the body to the probe.

\section{Master Haptic-Based Interface Device}

The haptic-based interface device integrates a SensAble PHANToM haptic system. This device has 6 dof and renders a 3D force information. It can track the position and orientation of the tool within a workspace of $16 \mathrm{~cm}$ wide, $13 \mathrm{~cm}$ high and $13 \mathrm{~cm}$ deep. The maximum exertable force is 6.4 Newton.

The project constraints on the master workstation site are mainly real time information feedback and geometric modelling of the remote patient.
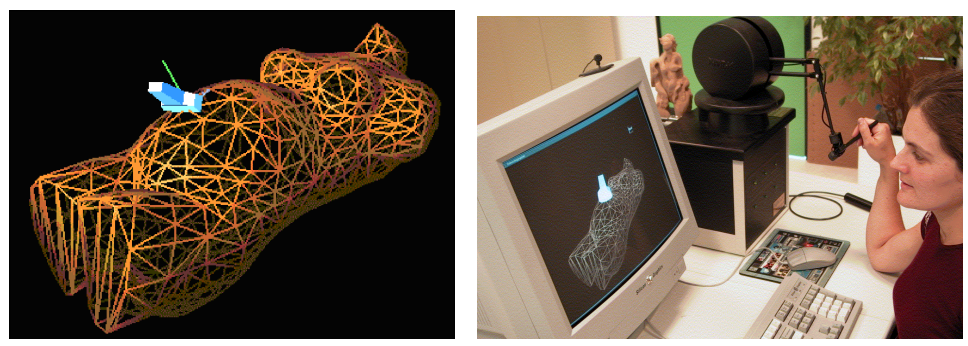

Fig. 2. Master interface device: (left) the local model and (right) the master haptic-based interface device on use

A 3D geometric model of the remote patient is coded with a polygonal mesh. The model is very useful to guarantee a force feedback at a fixed rate compatible with the gesture of the expert. The mesh results from the deformation of standard meshes already recorded and depending on the examination type. For a given patient, the mesh will be re-calculated thanks to registration from measurements on the patient (see [10] for more details). Remarkable points or surface data may be acquired before the exam using the slave robot with a process of tele-calibration ${ }^{1}$

The force feedback is calculated starting from a physical model of the virtual 3D shape. Force feedback computations are refreshed by measurements coming from the slave platform. In the model, the material point is heavy, elastic and viscous. The state of the material point is governed by mechanical equations. The virtual model of the patient is flexible and deformable and its mechanic characteristics - viscosity, stiffness, and mass - can be adjusted. The computation of forces from the contact location may be based (1) on the vertices of the 3D geometric model, or (2) according to the nearest polygon to the contact or (3) integrating its neighbourhood. The third solution is the best one. Experiments and results using this haptic control are encouraging and very realistic on the touch and feel level.

\footnotetext{
${ }^{1}$ Tele-calibration is a process making it possible to gauge an object or a distant $3 \mathrm{D}$ form with the help of the haptic flow.
} 


\section{The Slave Robot}

\section{Mechanical Architecture}

We can roughly decompose the echographic examination in two stages. One stage consists in exploring a large region of the body to localize the structure of interest. During these gross motions, control accuracy is not mandatory. In the second stage, the clinician locally explores the detected structure. These fine motions are mostly composed of rotations relatively to the contact surface of the probe. Therefore, it was reasonable to decouple translation and rotation dof. The TER slave robot is based on two parallel kinematic architectures and on artificial muscle actuation.

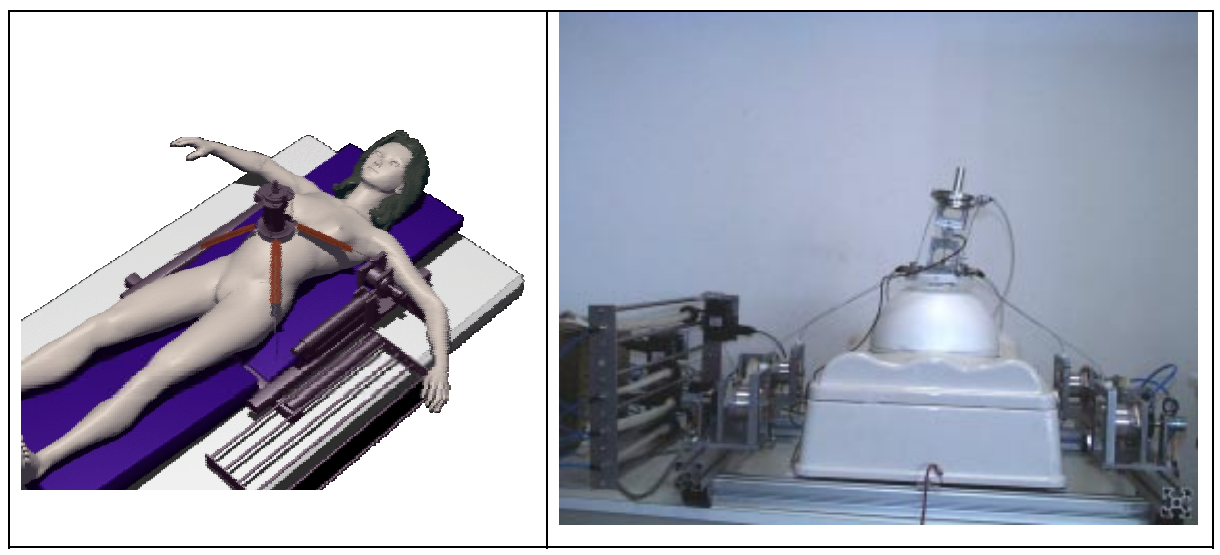

Fig. 3. The slave robot: (left) a sketch of the robot in use - (right) the prototype

As it can be seen on figure 3, a first parallel structure mounted on the consultation bed is composed of four antagonistic muscles enabling translations. They are connected to a ring supporting the probe and the other dof: this second parallel structure is also actuated by four muscles enabling the $3 \mathrm{D}$ orientation of the probe and fine translations. Both subsystems can be controlled simultaneously.

\section{Pneumatic Artificial Muscles}

The robot is entirely motorized with pneumatic artificial muscles commonly called McKibben artificial muscles. The McKibben muscle was invented in the 1950s to actuate pneumatic arm orthotics [11]; it was redesigned in the 1980s by Bridgestone engineers to obtain more powerful behaviour. The McKibben muscle now appears like one of the most original solution to give a robot a human-like joint softness which is not present in classical industrial robots, and consequently to develop "friendly robots" fully adapted to the new needs of service robotics [12].

The McKibben muscle is made of a thin rubber tube covered with a shell braided according to helical weaving as shown in figure 5. The muscle is closed by two ends, 
one being the air input and the other the force attachment point. When the inner tube is pressurized to a given pressure $\mathrm{P}$, the flexible pantograph network of the textile shell converts circumferential pressure forces into an axial contraction force which reduces the muscle length while its radius increases.
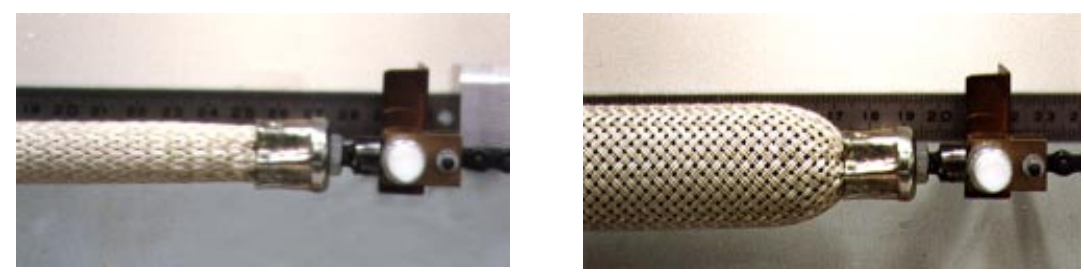

Fig. 4. McKibben muscle: (left) Initial contraction state, (right) maximum contraction state.

McKibben artificial muscles behave like a spring with a variable stiffness directly controlled by pressure $\mathrm{P}$, in analogy with the natural skeletal muscle controlled by the nervous activation as it is expressed in the classical tension-length diagram [13]. This spring character gives to the McKibben muscle its specific softness which can be called "natural compliance". The second aspect of the fundamental analogy between McKibben muscle and natural muscle is that in a given equilibrium position, it is possible to adapt the muscle force and the muscle stiffness by changing the control pressure. This double property of natural compliance and force/stiffness adaptation, combined with a closed-loop control in position or an hybrid position/force control, must lead to an accurate positioning of the probe while controlling the contact force on the body of the patient.

Up to now, the prototype has been designed and realized. It has been modelled and its kinematics has been simulated. Low level control schemes have been implemented for the muscles and tested. [14] reports these experiments.

\section{Communication}

\section{The Telecom Flows}

As can be seen on figure 1, different flows are necessary in the TER system. Each of these flows requires a specific transmission quality that will be discussed below.

The Visio phonic communication quality has to be as good as possible in order to facilitate the relationship between the patient and local operator and the expert. This includes: high quality bi-directional audio throughput and good transmission of the scene. Specific codec broad are used for this flow. The quality of the medical images that have to be transmitted depends on the phase of the exam: low quality is sufficient for the gross motion phase: high quality may be necessary for fine exploration; high quality is necessary for diagnosis and measurements. Compression algorithms based 
on wavelet transforms prove to be the best compromise between the image quality, the compression rate and the coding time (cf. [15]). The need for quality may be incompatible with the goal of low bandwidth network and indeed may restrict the application field. Specific codec broad are used for this flow on the remote site. The haptic control flow does not need high bandwidth but low end-to-end latency and very low variation of the latency. All these flows with their constraints have to be integrated in a single telecommunication channel. It is achieved by dedicated software that builds a specific telecommunication protocol in order to allow priority between flows.

The goal of low cost and widely usable system toward French healthcare centre led us to choose, in a first step, native ISDN networking. This telecommunication media provides good guaranty in term of bandwidth, stability of the latency, low latency. In a second step, Next Generation Internet will be considered.

\section{Tele Gesture}

We must take into account the physiological constraints of human gesture to make the tele-gesture possible. The haptic loop has to be fed at a frequency of $1 \mathrm{kHz}$ because below vibrations would be perceived making the gesture more difficult. We use the local model of the distant patient to calculate the force feedback at this rate. The network will feed the expert master interface device with real measurements at a lower rate. We experimented the transmission of haptic data on the ISDN network. These experiments were performed using two PHANToM systems communicating via the network. Each device sends its position and receives the position of the distant device. We tested the initialisation and shut down of the haptic session, the transmission of the position to the distant device, and the calculation of the efforts at $1 \mathrm{khz}$ with a data refresh at the reception with various rates ranging from 10 to $500 \mathrm{~ms}$. The first experimental results are reported in table 1.

Table 1. Transmission experiments using the ISDN network

\begin{tabular}{|l|l|l|l|l|}
\hline Sample (ms) & 10 & 100 & 250 & 500 \\
\hline Flow & $100 \mathrm{~Hz}$ & $10 \mathrm{~Hz}$ & $4 \mathrm{~Hz}$ & $2 \mathrm{~Hz}$ \\
\hline $\begin{array}{l}\text { Haptic be- } \\
\text { haviour }\end{array}$ & $\begin{array}{l}\text { Very } \\
\text { good }\end{array}$ & Good & Some vibrations & $\begin{array}{l}\text { Some vibrations and } \\
\text { oscillations }\end{array}$ \\
\hline $\begin{array}{l}\text { Command } \\
\text { ability }\end{array}$ & Natural & $\begin{array}{l}\text { Restricted in } \\
\text { term of the } \\
\text { gesture speed }\end{array}$ & Difficult & Very difficult \\
\hline Conclusion & Realistic & $\begin{array}{l}\text { Realistic for } \\
\text { slow gestures }\end{array}$ & $\begin{array}{l}\text { Need to set up } \\
\text { finer extrapolators } \\
\text { of gesture }\end{array}$ & $\begin{array}{l}\text { Need to set up finer } \\
\text { extrapolator and } \\
\text { stabilizer of gesture }\end{array}$ \\
\hline
\end{tabular}




\section{Conclusion}

In this paper, we have presented a system for tele-robotic echography. It combines a master station with or without force feedback with a slave robot which actuation through McKibben artificial muscles makes it naturally compliant. Some elements of the master station are operational. The slave robot has been designed and realized. Low-level control has been implemented. The tele-communication issues related to the transmission of gestures and images have been studied and experimented on an ISDN network. A lot remains to be done concerning system integration and evaluation before assessing that the objective of performing remote echography is reached; nevertheless we think that the development of original robotic architectures and tele-gesture capabilities are already very positive deliverables of such a project.

\section{Acknowledgements}

This project is supported by the French Ministry of Research and Technology (action line "ACI Telemédecine"), by France Telecom R\&D and by UAEM/CONACyT.

\section{References}

[1] on line http://www.irisi-nordpasdecalais.org/acercles/loginat.htm

[2] on line http://www.crcg.edu/projects/teleinvivo.html

[3] A. Gourdon, P. Vieyres, Ph. Poignet, M. Szpieg, Ph. Arbeille, "A tele-scanning robotic system using satellite", in EMBC99 Proceedings, Vienna, November 1999.

[4] A. Gourdon, Ph. Poignet, G. Poisson, P.Vieyres, P.Marché, "A new robotic mechanism for medical application, Proceedings ASME, Atlanta Georgia, September 1999.

[5] D. de Cunha, P. Gravez, C Leroy, E. Maillard, J. Jouan, P Varley, M Jones, M Halliwell, D. Hawkes, P. N. T. Wells, L. Angelini, "The MIDSTEP System for Ultrasound guided Remote Telesurgery", in Proceedings of 20th International Conference of the IEEE Engineering in Medicine and Biology Society. pp 1266 - 1269, 1998

[6] S.E. Salcudean, G. Bell, S. Bachmann, W.H. Zhu, P. Abolmaesumi, P.D. Lawrence, "Robot-assisted diagnostic ultrasound - design and feasibility experiments", in MOCCAI'99, C. Taylor and A. Colchester Eds., LNCS Series, Vol. 1679, pp1063-1071, Springer Verlag, 1999.

[7] Dombre, E., Thérond, X., Dégoulange, E., and Pierrot, E. Robot-Assisted detection of atheromatous plaques in arteries. In Proceedings of the IARP Workshop on Medical Robots, pp 133-140, Vienna, 1996

[8] Boudet, S., Gariepy, J., Mansour S. An Integrated Robotics and Medical Control Device to Quantify Atheromatous Plaques : Experiments on the Arteries of a Patient, in Proceedings of the $10^{\text {th }}$ IROS Conference, Grenoble, 1997

[9] Masuda K., Kimura E., Tateishi N., Ishihara K., Development of remote diagnosis system by using probe movable mechanism and transferring echogram via high speed digital network, in Proceedings of IXth Mediterranean Conference on Medical and Biological Engineering and Computing, MEDICON 2001, Croatia, June 2001 
[10] B. Couteau, Y. Payan, S. Lavallée, M.C. Hobatho, "The Mesh-matching algorithm : a new automatic 3D mesh generator for finite element analysis", in MICCAI'99, C. Taylor and A. Colchester Eds., LNCS Series, Vol. 1679, pp1175-1182, Springer Verlag, 1999.

[11] V.L. Nickel, M.D.J. Perry, and A.L. Garret, "Development of Useful Function in the Severely Paralysed Hand", The Journal of Bone and Joint Surgery, vol. 45-A, no. 5, pp. 933-952, 1963.

[12] B. Tondu and P. Lopez, "Modeling and Control of McKibben Artificial Muscle Robot Actuators", IEEE Control Systems Magazine, vol. 20, no.2, pp. 15-38, 2000.

[13] C. Ghez, "Muscles: Effectors of the Motor System", Chap. 36. in Principles of Neural Science, Kandel, E.R., Schwartz., J.H., Jessekk, T.M. (eds), Englewood Cliffs: PrenticeHall, pp. 548-563, 1991.

[14] A. Vilchis, J. Troccaz, P. Cinquin, F. Courrèges, G. Poisson, B. Tondu, "Robotic teleultrasound system (TER): slave robot control", in Proceedings of TA'2001, IFAC Conference on Telematics Application in Automation and Robotics, July 2001

[15] C. Delgorge, P. Vieyres, G. Poisson, C. Rosenberger, P. Arbeille: "Comparative survey of ultrasound images compression methods dedicated to a tele-echography robotic system", in Proceedings of the IEEE EMBS 2001 international conference, 2001 